ORIGINAL ARTICLE

\title{
The prognostic value of progesterone receptor status in meningiomas
}

\author{
F Roser, M Nakamura, M Bellinzona, S K Rosahl, H Ostertag, M Samii
}

J Clin Pathol 2004;57:1033-1037. doi: 10.1136/icp.2004.018333

See end of article for authors' affiliations .....................

Correspondence to: Dr F Roser, Department of Neurosurgery, University of Tübingen, Hoppe-Seyler Str.3, 72076, Tübingen, Germany; f.roser@gmx.de

Accepted for publication 21 April 2004
Background: Meningiomas are known to recur frequently, and their longterm management remains controversial. Previous studies indicate that progesterone and its receptors can play a role in the recurrence of meningiomas, but the correlation between the presence of these receptors and patients' outcome is unclear.

Aim: To conduct a retrospective analysis to investigate the prognostic relevance of progesterone receptor (PR) expression in meningiomas.

Methods: Five hundred and eighty eight meningiomas operated on over a period of 10 years were examined immunohistochemically to determine the PR status using monoclonal antibodies. Several factors including recurrence (mean follow up of 65 month), sex, tumour tissue consistency, location, vascularity, and en plaque appearance were analysed.

Results: PR status showed comparable values for men and women. World Health Organisation (WHO) grade II and III tumours had significantly fewer receptors than benign meningiomas. There was no significant correlation between PR status and recurrence rates in WHO grade I totally removed meningiomas. However, a combination of PR status and proliferation indices was shown to predict recurrence reliably.

Conclusions: Together with routine histological evaluation, PR status can help to describe the biological behaviour of meningiomas. Only a combination of clinical and biological features can describe the behaviour of meningiomas, predict their recurrence, and help to devise more effective follow up strategies.
M eningiomas are among the most common tumours of the central nervous system. They occur with a frequency of $19 \%$ at intracranial and $25 \%$ at spinal localisations. ${ }^{1}$ One of the accepted prognostic indicators of this tumour entity is the World Health Organisation (WHO) grading system, which distinguishes three grades of malignancy. ${ }^{2}$ Because the growth potential of meningiomas is variable, and even the WHO microscopic morphological classification frequently fails to predict their clinical behaviour, quantifying the hormonal status of the tumour may help to predict its biological behaviour and provide options for further treatments.

In the past two decades, the relation between sex hormone receptors and meningiomas has been the subject of several studies. $^{3-9}$ The higher incidence of meningiomas among women, their behaviour during pregnancy, and the reported epidemiological link between meningiomas and breast carcinomas have led to the assumption that sex steroid hormones may influence the growth of meningiomas. ${ }^{10-12}$ Supported by promising results in breast cancer treatment, new chemotherapeutic approaches based on hormone manipulation were tested in patients with meningioma. ${ }^{13} 14$

\section{"There are several reports suggesting that a positive progesterone receptor status is linked to later recurrence"}

In recent years, the availability of highly specific monoclonal antibodies has provided a rapid and reliable method to assess the receptor status in meningiomas. It is generally agreed that most meningiomas express PRs but are devoid of oestrogen receptors. ${ }^{815}$ Nearly all previous investigators reported that the presence of PRs in meningiomas does not correlate with patients' age or sex, or with the location or size of the tumours. ${ }^{6}{ }^{17}$ Several studies also reported a decrease in
PR expression from low grade to high grade meningiomas. No correlation between PR status and histological subtype has been reported, with the exception of two studies showing the increased presence of PRs in the meningotheliomatous type. ${ }^{417} 18$

There are several reports suggesting that a positive PR status is linked to later recurrence. ${ }^{4}{ }^{6}$ However, these studies were based on small and non-homogeneous patient populations. To evaluate the prognostic value of PR expression in meningiomas, our patients group was defined according to stringent selection criteria and was large enough to allow reliable statistical analysis.

\section{MATERIAL AND METHODS \\ Patient population}

Five hundred and eighty eight paraffin wax blocks from 554 patients with intracranial meningiomas, operated on at the neurosurgical department, Klinikum Hannover Nordstadt, Germany, between 1990 and 2000 were randomly retrieved from the archives of the department of pathology. All of the cases used in our report were included in a previous study on proliferative indices using Ki-67 antibody in meningiomas. ${ }^{19}$

There were 192 male and 396 female patients (ratio $1: 2$ ), whose age ranged from 12 to 93 (mean, 53.3) years. Nine per cent of the patients were lost to follow up, the others were followed for a median of 63 months (range, 3-228) or until death. The material included samples from 20 patients with neurofibromatosis type 2 (NF-II; total of 27 tumours), who had a mean age of 32.3 years at presentation (range, 16-60) and a male to female ratio of $1: 1$. Clinical information about each patient was obtained through review of medical records,

Abbreviations: IRS, immunoreactive score; LI, labelling index; NF-II, neurofibromatosis type 2; PR, progesterone receptor; WHO, World' Health Organisation 


\begin{tabular}{ccc} 
Table 1 & PR status in meningiomas \\
\hline \multicolumn{4}{l}{ No. of cases } & PR + (in \%) \\
\hline WHO I & 533 & $212(56.5 \%)$ \\
Female & 375 & $96(60.7 \%)$ \\
Male & 158 & \\
WHO II & 46 & $8(44.4 \%)$ \\
Female & 18 & $13(46.4 \%)$ \\
Male & 28 & $1(33.3 \%)$ \\
WHO III & 9 & $0 \%$ \\
Female & 3 & \\
Male & 6 &
\end{tabular}

PR, progesterone receptor; WHO, World Health Organisation.

in addition to follow up examinations with clinical and neuroradiological evaluation, or through detailed questionnaires, including radiological reports of the latest magnetic resonance imaging scan, which was performed on a yearly basis after surgery. We defined recurrence as radiological appearance of new tumour masses at the site of origin of the initial meningioma.

Paraffin wax embedded sections were stained with haematoxylin and eosin. Meningiomas were categorised into subtypes according to the new WHO classification. ${ }^{2}$

Immunohistochemistry was performed using $2 \mu \mathrm{m}$ thick paraffin wax sections. In short, paraffin wax sections were dewaxed and stained with monoclonal mouse antihuman PR lA6 purchased from Dako (Copenhagen, Denmark), according to standard protocols using the ChemMate alkaline phosphatase detection kit from Dako and chromogen red as chromogen. Sections were counterstained with haematoxylin and coverslipped with KP tape (KliniPath BV, Duiven, the Netherlands). Negative controls were run at each staining session, as were positive control specimens using breast cancer tissue (cell line MB-CCREZ). In each case, the entire section was systematically examined using an optical grid at high power $(\times 400$; Zeiss microscope $)$ for the presence of immunoreactivity. All slides were examined for positively stained tumour cell nuclei regardless of tumour grade. The receptor status was determined by a semiquantitative scoring scale with respect to staining intensity (graded as: 0, absent; 1, weak; 2, moderate; and 3, strong) and percentage of positive tumour cells $(0$, indicating the absence of positive nuclei; 1 , the presence of a few positive tumour nuclei $<10 \%$ in the entire section; 2 , an estimated $10-50 \%$ positive nuclei; $3,51-80 \%$ positive tumour nuclei; and $4,>80 \%$ positive tumour nuclei). As recommended for breast cancer, ${ }^{20}$ and verified with meningioma tissue, ${ }^{15}$ an immunoreactive score (IRS) was calculated for each tumour by multiplying the staining intensity by the indicator for positive tumour cells, producing an IRS range from 0 to 12 . Tumours with an IRS of 2 or more were considered receptor positive. Only unquestionably stained nuclei were accepted as positively stained. Randomly chosen slides were counted by independent researchers to evaluate interindividual variability. However, no significant differences in PR positivity judgement were noted. Vascularity was assessed semiquantitatively and reflects the neurosurgeon's impression at the time of surgery. A scale from 0 to 4 was used to indicate low to high vascularisation of the operated meningioma.

Statistical analysis was performed using the SPSS 11.0 (SPSS Inc) software program for Windows. Several parameters shown in the result section were analysed by means of the Student's $t$ test or log rank tests. Mean value, SD, and $\mathrm{p}$ value were calculated. Differences were considered significant at a $\mathrm{p}$ value of $<0.05$. Multivariate analysis was performed by means of ANOVA to determine the significance of different variables. The distribution of the recurrence free survival times was estimated using the Kaplan-Meier

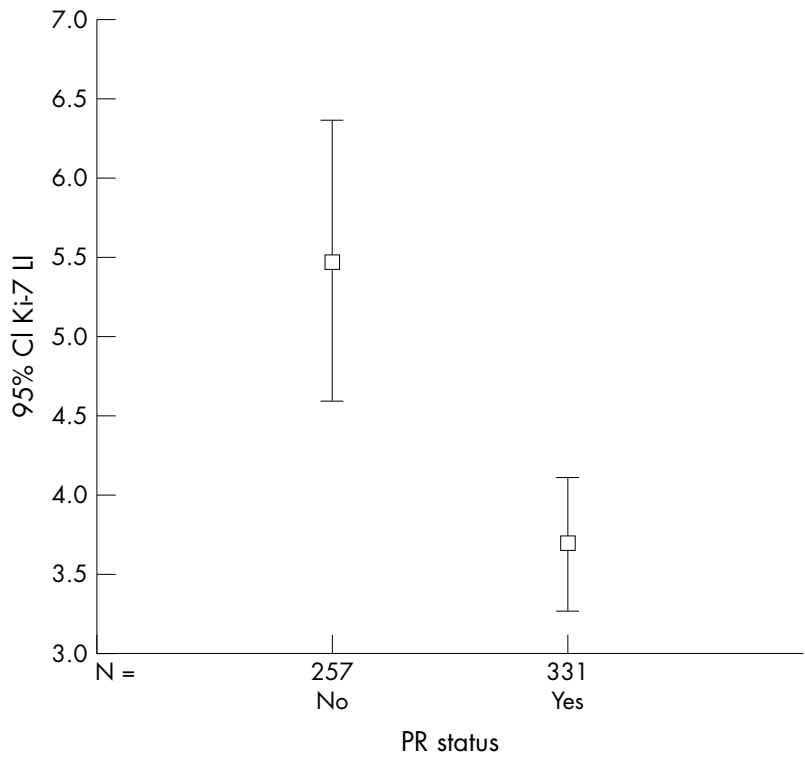

Figure 1 Correlation between proliferative index and progesterone receptor (PR) status. Ki-67 labelling index (LI) correlated with PR status. Mean of $5.48 \%$ (PR negative) versus 3.69\% (PR positive); $p<0.0001$. $\mathrm{Cl}$, confidence interval.

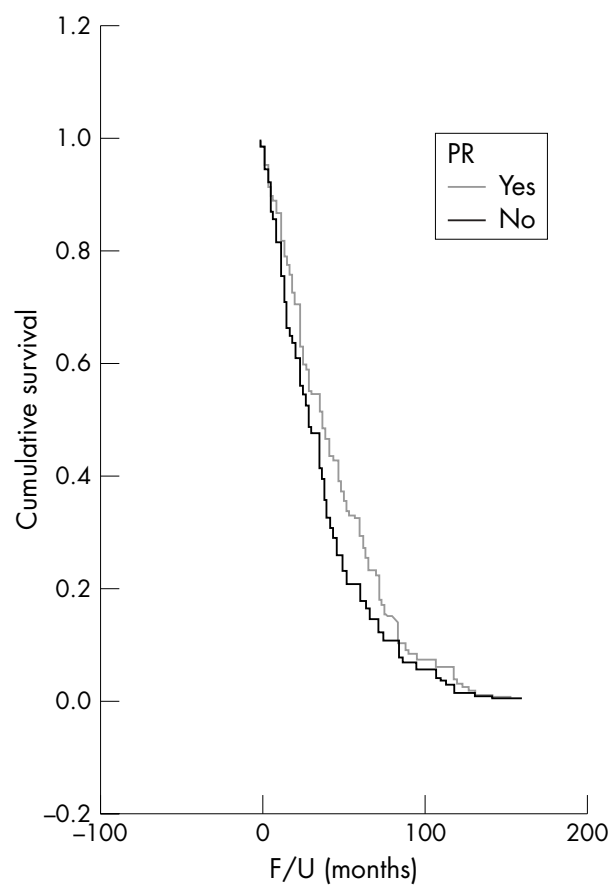

Figure 2 Recurrence free survival. I. Kaplan-Meyer curves analysing only World Health Organisation grade I and Simpson grade I cases, with neurofibromatosis type 2 cases excluded. F/U, follow up; PR, progesterone receptor.

method. Pearson's regression analysis was performed to determine the correlation between PR status and other influencing factors. Recurrence was defined as radiologically detected evidence of regrowth regardless of symptoms.

\section{RESULTS}

The PR status was positive in 111 of the 192 male $(57.8 \%)$ and in 218 of the 396 female patients (55.1\%). The mean age of the patients with a positive PR status was 55.4 years for 
females (range, 17-83; SD, 12.16) and 51.5 years for males (range, 15-77; SD, 14.18). These differences were not significant. In addition, there was no higher PR positivity in younger patients ( $<37$ years: mean PR positivity, $45.3 \%$ ) or in patients with NF-II (mean PR positivity, 48.2\%). There was significantly lower PR expression in WHO grade II (atypical) and III (anaplastic) meningiomas, compared with WHO grade I meningiomas (table $1 ; p<0.0001$ ). There was a significant difference in the PR status between the meningotheliomatous ( $\mathrm{n}=376$; $\mathrm{PR}$ positivity in $64.6 \%$ ) and fibrous subtypes $(\mathrm{n}=77 ; \mathrm{PR}$ positivity in $31.1 \%$; $\mathrm{p}<0.0001)$. The PR status differences between the other subtypes-54 transitional (PR positivity, 50.0\%), 10 psammomatous (PR positivity, 60.0\%), six angiomatous (PR positivity, 66.6\%), seven clear cell (PR positivity, 40.0\%), and five microcystic meningiomas (PR positivity, 60.0\%)did not reach significance. There was also no significant association between PR status and tumour location $(\mathrm{p}=0.273)$.

Patients with first time treated meningiomas $(n=491)$ were compared with those with a local recurrence of a previously operated meningioma $(n=97)$. Of all first time treated meningiomas, $53.5 \%$ were $\mathrm{PR}$ positive, compared with $46 \%$ of recurrent meningiomas ( $p<0.0001$ ), although the tumours did not show a different grade of malignancy according to the WHO grading at the time of recurrence.

The proliferation rate determined by the Ki- 67 labelling index (LI) showed a mean index of $4.47 \%$ (SD, $5.71 \%$; range, $0-85 \%$ ); high vascularity (grade 4 ) was seen in 86 cases.

Among these parameters, male sex, increased vascularity, and high PR status were significantly related to increased time to recurrence (ANOVA; p < 0.0005). Increased vascularity was associated with significant increases in progesterone staining and decreases in the WHO index and Ki-67 LI. In addition, an increased WHO index was associated with decreased PR status and increased Ki-67 LI. Finally, decreased progesterone staining was associated with increased Ki-67 LI ( $\mathrm{n}=$ 555; $\mathrm{p}<0.001$; fig 1 ).

The dependence of time to recurrence on resection grade was analysed. For this survival analysis, WHO grade II and III meningiomas were excluded and all WHO grade I meningiomas with recurrences $(n=120)$ were separated into four different resection grades according to the Simpson classification. For WHO grade I and Simpson grade I resected meningiomas there was no significant correlation between recurrence free survival and PR status (fig 2; $p=0.795$ ).

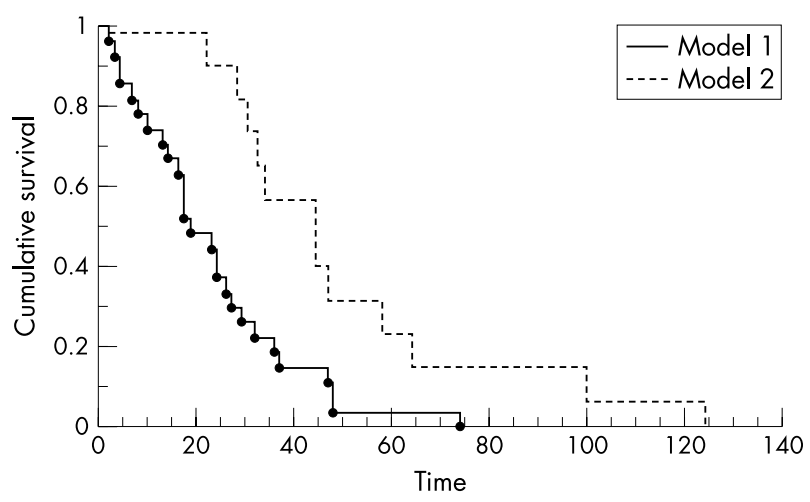

Figure 3 Recurrence free survival. II. Kaplan-Meyer cumulative plot. Hazard plot for new recurrence. A model of two prognostic factors considering prognosis in totally removed meningiomas (Simpson I + II). Time scale in months. Model 1: PR status negative or score $<2+$ and $\mathrm{Ki}-67 \mathrm{LI} \geqslant 4 \%$. Model 2: PR status positive or score $\geqslant 2+$ and $\mathrm{Ki}-67$ $\mathrm{LI}<4 \%$. LI, labelling index; PR, progesterone receptor.
Combining two factors of proposed prognostic importance in benign meningiomas - the proliferation index (Ki-67 LI, $<4 \% v \geqslant 4 \%$ ) and PR status (negative $v$ positive)-a significantly decreased recurrence free survival could be shown for negative PR status and Ki-67 LI $\geqslant 4 \%$ (fig 3; $\mathrm{p}=0.002$ ).

\section{DISCUSSION}

Even after complete removal according to the Simpson classification, WHO grade I meningiomas frequently recur. ${ }^{21-23}$ The role of steroid hormones in the progression of meningiomas is still a matter of controversy. Several studies, which included meningiomas of all grades, suggested a more favourable prognosis for PR positive meningiomas. ${ }^{3} 152425$ However, these studies frequently included meningiomas with different resection grades and histological subtypes (table 2).

The aim of our study was to test whether the PR status itself is related to recurrence free survival in patients with meningioma. For the group of patients to be homogeneous, atypical and anaplastic meningiomas, in addition to incompletely resected tumours with dural infiltration, were excluded from the survival analysis. Testing for the validity of a predictive recurrence marker would be compromised if both subtotally and radically removed tumours were incorporated in the same statistical analysis, because the extent of resection is the most influential factor in recurrence. ${ }^{123} \mathrm{~A}$ thorough review of the literature provides several studies suggesting a significant relation between PR status and recurrence free survival (table 2). However, when we used the above mentioned method of analysing a homogeneous group of patients, our results differed from those already published. ${ }^{3} 4624$

\section{"The focus of interest should be benign borderline cases where additional information is needed for prognostic considerations"}

Using these stringent criteria, our results showed no significant correlation between the PR status alone and recurrence free survival in patients with benign meningioma after radical tumour resection (fig 2). Hsu and colleagues suggested that only a combination of three proposed prognostic factors for survival-that is, WHO grade, proliferative index, and PR status-should be used to predict meningioma recurrence. ${ }^{24}$ However, their model was tested on a sample containing atypical and malignant meningiomas, and therefore did not demonstrate the true influence of PR status on survival. The focus of interest should be benign borderline cases where additional information is needed for prognostic considerations. We studied prognostic factors among benign meningiomas and found PR status effected survival only in combination with the proliferation marker Ki-67 (fig 3).

Cahill et al and Brandis et al showed that malignant meningiomas are devoid of PR and oestrogen receptors. ${ }^{57152627}$ Furthermore Whittle et al reported that PR negative meningiomas were biologically more aggressive than PR positive ones. ${ }^{25}$ Our data confirmed the presence of significantly higher $\mathrm{PR}$ values in benign meningiomas compared with WHO grade II or III tumours.

Although not entirely unlikely, no association between PR status and variables such as age, tumour location, first time resection versus reoperation, and histological subtype has been reported in the literature so far. Statistical analysis in our present study was conducted to confirm this and revealed no significant associations between the above mentioned factors and PR status. This is in accordance with the available 
Table 2 Review of the literature

\begin{tabular}{|c|c|c|c|c|c|}
\hline Reference & No. patients & WHO I & Simpson I & Follow up time (months) & RFS (p value) \\
\hline Present study & 555 & 526 & 262 & 63 & 0.795 \\
\hline Strik et al $(2002)^{4}$ & 93 & 93 & 93 & 180 & 0.01 \\
\hline Gursan et al $(2002)^{3}$ & 110 & 105 & NA & NA & Significant \\
\hline Fewings et al $(2000)^{6}$ & 62 & 60 & 20 & 84 & 0.013 \\
\hline Hsu et al $(1997)^{24}$ & 70 & 28 & NA & 65 & 0.0001 \\
\hline
\end{tabular}

literature, ${ }^{6} 162829$ although some studies suggest a worse prognosis for meningotheliomatous type tumours. ${ }^{24} 3031$ In our study, the fibrous meningioma subtype showed significantly lower PR expression, although there was no correlation with prognosis.

All available data in the literature point to higher expression of PR in female patients. ${ }^{3615242632}$ However, these studies included a substantial number of atypical and malignant meningiomas. These meningiomas are mostly devoid of PRs and are more often found among male patients. After excluding atypical and malignant cases, no sex related difference in PR expression could be found. Therefore, the previously reported sex related difference might be the result of particular selection criteria, which produced a non-homogeneous patient population.

Previous studies on the human endometrium have shown that progesterone plays a role in neovasculogenesis. High expression of the PR in meningiomas may indicate a similar situation. However, Fewings et al suggested that progesterone may inhibit angiogenesis, by enhancing the production of thrombospondin 1, an angiogenesis inhibitor. ${ }^{6}$ In our series, there was a correlation between high vascularity and positive PR status. However, it is difficult to draw conclusions from these data, because most PRs in meningiomas are nonfunctional..$^{5}$ Moreover, our observations come from a clinical and histopathological analysis and may well reflect a multifactorial situation, with progesterone being only one variable in the process of tumour vascularisation. It should again be pointed out that the assessment of vascularity was semiquantitative and reflected the neurosurgeon's impression at the time of surgery. Therefore, only further immunohistological studies could reliably show how PRs may influence tumour vascularity in meningiomas.

Markwalder et al and Perrot-Applanat et al found no correlation between proliferation and PR status. ${ }^{81} 33$ Other authors and our present data showed a strong correlation between high Ki-67 LI and negative PR status. ${ }^{37}$ The correlation was even more significant among female patients. We have shown previously that proliferation index and PR status in combination are important prognostic factors in meningiomas. ${ }^{19}$

\section{Take home messages}

- Progesterone receptor (PR) status alone cannot be used to predict prognosis in meningiomas

- However, PR status in combination with the proliferative index can be a useful prognostic tool for benign meningiomas

- In histopathological borderline cases, the PR status, combined with the routine pathological investigation can provide more insight in the behaviour of a meningioma, particularly in the presence of a high proliferation index, subtotal resection, or recurrence
At present, it is still unclear whether areas of PR positivity in meningiomas reflect the status of the whole tumour. PR positive cells in all histological grades are distributed heterogeneously throughout the tumour..$^{15} 34$ Because of modern microsurgical techniques (ultrasound aspiration), where only tumour parts with no precisely named location within the tumour reach the pathologist, focal accumulation of biological activity within the meningioma may be missed or misjudged..$^{35}$ This can account for different results among different laboratories, or even within one tumour.

The results of our present study suggest that PR status in combination with the proliferative index can be a useful prognostic tool for benign meningiomas. In histopathological borderline cases, the PR status, combined with the routine pathological investigation, can provide more insight in the behaviour of a meningioma, particularly in the presence of a high proliferation index, subtotal resection, or recurrence.

However, the PR status alone cannot be used to predict behaviour in benign meningiomas, and should not influence the decision about follow up intervals and therapeutic strategies. Moreover Matsuda et al showed that the antitumorous effect of antiprogesterone agents on meningiomas, both in vitro and in vivo, exists regardless of the PR status of the tumour, suggesting that the antitumorous effect is mediated by other pathways. ${ }^{36}{ }^{37}$ This was supported by Verheijen and co-workers, who suggested that PR-like proteins do not have a biological function and may lead to overestimation of the PR status for clinical purposes. ${ }^{36}$

\section{Authors' affiliations}

F Roser, Department of Neurosurgery, University of Tübingen, 72076, Tübingen, Germany

M Nakamura, M Bellinzona, Department of Neurosurgery, Klinikum Hannover Nordstadt, 30167 Hannover, Germany

S K Rosahl, Department of Neurosurgery, University of Freiburg, 79106 Freiburg, Germany

H Ostertag, Institute of Pathology, Klinikum Hannover Nordstadt M Samii, International Neuroscience Institute, 30625 Hannover, Germany

\section{REFERENCES}

1 Jaaskelainen J. Seemingly complete removal of histologically benign intracranial meningioma: late recurrence rate and factors predicting recurrence in 657 patients. Surg Neurol 1986;26:461-9.

2 Kleihues P, Burger PC, Scheithauer B. The new WHO classification of brain tumors. Brain Pathol 1993;3:255-68.

3 Gursan N, Gundogdu C, Albayrak A, et al. Immunohistochemical detection of progesterone receptors and the correlation with $\mathrm{Ki}-67$ labeling indices in paraffin-embedded sections of meningiomas. Int J Neurosci 2002;1 12:463-70.

4 Strik HM, Strobelt I, Pietsch-Breiffeld B, et al. The impact of progesterone receptor expression on relapse in the long-term clinical course of 93 benign meningiomas. In Vivo 2002;16:265-70.

5 Blankenstein MA, Verheijen FM, Jacobs JM, et al. Occurrence, regulation, and significance of progesterone receptors in human meningioma. Steroids 2000;65:795-800

6 Fewings PE, Battersby RD, Timperley WR. Long-term follow up of progesterone receptor status in benign meningioma: a prognostic indicator of recurrence? J Neurosurg 2000;92:401-5.

7 Nagashima G, Aoyagi $M$, Wakimoto $\mathrm{H}$, et al. Immunohistochemical detection of progesterone receptors and the correlation with $\mathrm{Ki}-67$ labeling indices in paraffin-embedded sections of meningiomas. Neurosurgery 1995;37:478-82 
8 Perrot-Applanat M, Groyer-Picard MT, Kujas M. Immunocytochemical study of progesterone receptor in human meningioma. Acta Neurochir (Wien) $1992 ; 115: 20-30$

9 Markwalder TM, Gerber HA, Waelti E, et al. Hormonotherapy of meningiomas with medroxyprogesterone acetate. Immunohistochemical demonstration of the effect of medroxyprogesterone acetate on growth fractions of meningioma cells using the monoclonal antibody Ki-67. Surg Neurol 1988;30:97-101.

10 Bonito D, Giarelli L, Falconieri G, et al. Association of breast cancer and meningioma. Report of 12 new cases and review of the literature. Pathol Res Pract 1993;189:399-404.

11 Lieu AS, Hwang SL, Howng SL. Intracranial meningioma and breast cancer. J Clin Neurosci 2003;10:553-6.

12 Michelsen JJ, New PF. Brain tumour and pregnancy. J Neurol Neurosurg Psychiatry 1969;32:305-7.

13 Grunberg SM. Role of antiprogestational therapy for meningiomas. Hum Reprod 1994;9(suppl 1):202-7.

14 Lamberts SW, Tanghe HL, Avezaat CJ, et al. Mifepristone (RU 486) treatment of meningiomas. J Neurol Neurosurg Psychiatry 1992;55:486-90.

15 Brandis A, Mirzai S, Tatagiba M, et al. Immunohistochemical detection of female sex hormone receptors in meningiomas: correlation with clinical and histological features. Neurosurgery 1993;33:212-17.

16 Schrell UM, Adams EF, Fahlbusch R, et al. Hormonal dependency of cerebral meningiomas. Part 1: female sex steroid receptors and their significance as specific markers for adjuvant medical therapy. J Neurosurg 1990;73:743-9.

17 Ironside JW, Battersby RD, Dangerfield VJ, et al. Progesterone receptors in meningiomas: morphometric assessment of vascularity and cellularity on nea facsimile cryostat sections [letter]. J Clin Pathol 1986;39:810-11.

18 Gabos S, Berkel J. Meta-analysis of progestin and estrogen receptors in human meningiomas. Neuroepidemiology 1992;11:255-60.

19 Roser F, Samii M, Ostertag H, et al. The Ki-67 proliferation antigen in meningiomas. Experience in 600 cases. Acta Neurochir (Wien) $2004 ; 146: 37-44$

20 Remmele W, Stegner HE. [Recommendation for uniform definition of an immunoreactive score (IRS) for immunohistochemical estrogen receptor detection (ER-ICA) in breast cancer tissue.] Pathologe 1987;8:138-40.

21 Nakasu S, Nakasu Y, Matsuda M. Meningioma recurrence. J Neurosurg 2000;92:897-9

22 Yamasaki F, Yoshioka H, Hama S, et al. Recurrence of meningiomas. Cancer 2000;89: 1 102-10.
23 Simpson D. The recurrence of intracranial meningiomas after surgical treatment. J Neurol Neurosurg Psychiatry 1957;20:22-39.

24 Hsu DW, Efird JT, Hedley-Whyte ET. Progesterone and estrogen receptors in meningiomas: prognostic considerations. J Neurosurg 1997;86:113-20.

25 Whittle IR, Foo MS, Besser M, et al. Progesterone and oestrogen receptors in meningiomas: biochemical and clinicopathological considerations. Aust N Z J Surg 1984;54:325-30.

26 Cahill DW, Bashirelahi N, Solomon LW, et al. Estrogen and progesterone receptors in meningiomas. J Neurosurg 1984;60:985-93.

27 Magdelenat H, Pertuiset BF, Poisson M, et al. Progestin and oestrogen receptors in meningiomas. Biochemical characterization, clinical and pathological correlations in 42 cases. Acta Neurochir (Wien) 1982;64:199-213.

28 Hsu DW, Pardo FS, Efird JT, et al. Prognostic significance of proliferative indices in meningiomas. J Neuropathol Exp Neurol 1994;53:247-55.

29 Khalid H. Immunohistochemical study of estrogen receptor-related antigen, progesterone and estrogen receptors in human intracranial meningiomas. Cancer 1994;74:679-85.

30 Horsfall DJ, Goldsmith KG, Ricciardelli C, et al. Steroid hormone and epidermal growth factor receptors in meningiomas. Aust N Z J Surg 1989:59:881-8.

31 Markwalder TM, Zava DT, Goldhirsch A, et al. Estrogen and progesterone receptors in meningiomas in relation to clinical and pathologic features. Surg Neurol 1983:20:42-7.

32 Carroll RS, Glowacka D, Dashner K, et al. Progesterone receptor expression in meningiomas. Cancer Res 1993;53:1312-16.

33 Markwalder TM, Markwalder RV, Zava DT. Estrogen and progestin receptors in meningiomas: clinicopathological correlations. Clin Neuropharmacol 1984;7:368-74.

34 Ohta M, lwaki T, Kitamoto T, et al. MIB1 staining index and scoring of histologic features in meningioma. Indicators for the prediction of biologic potential and postoperative management. Cancer 1994;74:3176-89.

35 Nakasu S, Li DH, Okabe H, et al. Significance of MIB-1 staining indices in meningiomas: comparison of two counting methods. Am J Surg Pathol 2001;25:472-8.

36 Verheijen FM, Donker GH, Viera CS, et al. Progesterone receptor, bc1-2 and bax expression in meningiomas. J Neurooncol 2002;56:35-41.

37 Matsuda Y, Kawamoto K, Kiya K, et al. Antitumor effects of antiprogesterones on human meningioma cells in vitro and in vivo. J Neurosurg 1994;80:527-34. 